\title{
Twin Registries: An Ongoing Success Story
}

\author{
Andreas Busjahn,' and Yoon-Mi Hur ${ }^{2}$ \\ ' HealthTwiSt GmbH, Berlin, Germany \\ ${ }^{2}$ Medical Research Center, Seoul National University, Seoul, South Korea
}

In the mid-1990s a German funding agency rejected a grant application for a twin heritability and linkage study on the grounds that 'twin studies were an outdated methodology with no scientific merit, publishable only in obscure journals if anything ...' Neither Twin Research and Human Genetics nor any of the other peer-reviewed journals cited by the authors of this special issue warrants the term 'obscure'. Rather than being 'outdated', twin research has proven its increasing value for studying the complex interplay of genes and environment in humans; PubMed lists 713 entries for 'twins' for 1990, this number increasing to 723, 979 and 1126 in 1995, 2000 and 2005, respectively.

One of the fundamental requirements for twin studies is access to twins, and twin registries, rather than spontaneous recruitment on a 'per study' basis, is the way to achieve this. In 2002 this journal devoted a full special issue to this topic, and established as well as newly emerging twin registries presented their recruitment strategies, phenotyping focus and their visions for future research and collaborations. The purpose of that issue was not just to serve as a directory of registries but was to encourage others to start or extend twin collections without having to 'reinvent the wheel'.

Since 2002, significant progress has been made around the globe. New twin registries have emerged in countries including Chile, Israel, Spain, and
Taiwan, while others have been founded in the United States (US) and Europe, reflecting the worldwide acceptance of the twin registry as a valuable resource for scientific research. In addition, twin registries have been increasingly involved in various fields of science, resulting in the creation of innovative research projects. This special issue introduces a variety of research areas using registries of twins, ranging from prenatal to adulthood in age, which, we believe, will significantly contribute to the future advance of science.

Whereas 36 registries were listed in 2002, the number has increased to 52 in this issue. We are grateful to the contributors for well-prepared manuscripts, presenting recent and novel findings and stimulating information and insights. In addition we are most appreciative of funding from the US National Institute of Environmental Health Sciences (NIEHS) without which this special issue would not have been possible. We also thank a number of authors who made contributions or paid full-page charges to help cover our expenses for this issue. Finally, we thank Nick Martin who encouraged us to produce this issue, and Marisa Grimmer for her expert editorial support. We hope that this special issue will serve as a useful resource for researchers from various scientific fields and facilitate international collaborations for the resolution of important research questions on human diseases and biobehavioral traits. 
Twin Research and Human Genetics December 2006

https://doi.org/10.1375/twin.9.6.705 Published online by Cambridge University Press 